\title{
Elastic modes of vortex configurations in thin disks
}

\author{
L. R. E. Cabral* and F. M. Peeters ${ }^{\dagger}$ \\ Departement Fysica, Universiteit Antwerpen (Campus Drie Eiken), Universiteitsplein 1, B-2610 Antwerpen, Belgium
}

(Received 1 September 2004; published 23 December 2004)

\begin{abstract}
Within the London limit, we investigate the dynamics of stable vortex configurations in small thin superconducting disks by studying their elastic modes. The elastic modes are a mixture of shearlike and compressionlike modes. More pronounced shearlike behavior is found for the low-energy eigenmodes, while a compressionlike content is more prominent for higher eigenmodes. The eigenmode associated with a pure rotation of the entire system is always zero. Highly symmetric stable configurations - in which the number of vortices in different shells are multiples of each other — have several degenerate modes, in contrast with nonsymmetric configurations, which have a less degenerate spectrum. The second lowest-energy mode is nearly zero in configurations of the latter type, while is clearly nonzero for highly symmetric configurations. This suggests that symmetric configurations are more stable against fluctuations, which is important in the development of new superconducting devices. The present work could also be applied to electric charges confined within a cylindrical hole inside a metal and submitted to a parabolic electric potential.
\end{abstract}

DOI: $10.1103 /$ PhysRevB.70.214522

PACS number(s): 74.20.De, 74.25.Dw, 74.25.Ha

\section{INTRODUCTION}

Vortex dynamics in superconductors has received much attention due to its importance in potential superconducting devices. In most of those applications, vortex motion is, in fact, an undesired feature, since it generates dissipation. Therefore, to understand the mechanisms which drive, prevent, and guide vortex motion is essential for applications of superconducting devices.

Vortex motion may be driven by thermal or quantum fluctuations. As a result, vortices randomly wander around their stable positions. Several consequences of such fluctuations in the vortex positions have been investigated. For example, at a given temperature, $T_{m}<T_{c}$, vortex lattice melting was investigated theoretically, ${ }^{1,2}$ numerically, ${ }^{3,4}$ and experimentally. ${ }^{5,6}$ Vortex fluctuations also induce modifications in the magnetization $(M),{ }^{7}$ which leads to a unique crossing point in the $M-T$ curves. $^{8}$ To investigate the influence of fluctuations on the vortex lattice it is important to know the elastic properties of such a lattice. ${ }^{9}$ It was shown that the elastic properties of the vortex lattice ${ }^{10,11}$ in bulk superconductors exhibit shear, compression, and tilt. In thin films there is no tilt of the vortex lattice. This elastic description has been shown to be important in the understanding of the behavior of the vortex lattice in the presence of fluctuations and pinning (see, for example, Refs. 12 and 13).

In mesoscopic superconductors, vortices have stable positions dictated by their mutual interaction and the geometry of the sample. ${ }^{14-18}$ The sample geometry acts as a kind of confinement potential (since the boundary determines the shielding current pattern and the appearance of "vortex images"14), which makes these systems resemble charged particles confined by some potential, ${ }^{19-21}$ at least for fields much smaller than $H_{c 2}$. In the latter system, the normal modes were used to investigate the melting temperature and the specific heat of charged particle clusters. ${ }^{19}$

In the present paper, we studied the elastic modes of the vortex configurations in small thin disks - i.e., in a finite system. These elastic modes are related to the elastic constants of a given vortex configuration. ${ }^{9}$ This can be understood by expanding the energy of the system close to a stable configuration,

$$
\mathcal{G}=\mathcal{G}_{0}+\sum_{i, \alpha} \frac{\partial \mathcal{G}}{\partial \rho_{i, \alpha}} \delta \rho_{i, \alpha}+\frac{1}{2} \sum_{i, \alpha} \sum_{j, \beta} \frac{\partial^{2} \mathcal{G}}{\partial \rho_{i, \alpha} \partial \rho_{j, \beta}} \delta \rho_{i, \alpha} \delta \rho_{j, \beta}
$$

where higher-order terms were omitted. Here $\rho_{i}$ is the [twodimensional (2D)] position of the vortex $i$. The italic symbols refer to vortex indices and the greek ones to the coordinates $x$ and $y$. The derivatives are to be evaluated at the vortex positions of a given stable configuration. The first derivatives are just the forces acting on each vortex, which are zero for a stable configuration. $\mathcal{G}_{0}$ is the energy of the stable configuration and, thus, $\mathcal{G}-\mathcal{G}_{0}$ represents the energy associated with the elastic displacement of the particles in the system. Thus, the second derivatives give the elements of the elastic tensor, which can be related to an effective elastic constant for each elastic mode and, consequently, to the energy required to excite such modes. One may also consider a simple physical picture for the present problem in which vortices would be connected to each other by springs. A similar model has been used to treat vortices in a periodic array of traps, the results of which were compared to numerical simulations. ${ }^{22,23}$

An important point worth to be mentioned is that, as vortices belong to the class of viscous systems, the normal modes are related to the dissipated power due to vortex motion. For example, if vortices are driven by an external force, the motion of the vortex configuration will be associated with a linear combination of the elastic modes, each of which with an exponential decay associated with its eigenvalue. It is also important to notice that, as pointed out in Refs. 14 and 24 and within the London approach, a vortex system may be regarded as charged particles if the penetration length ( $\lambda$ for bulk or $\Lambda=\lambda^{2} / d$ for thin film superconductors) is much 
larger than the typical dimensions of the system. Therefore, the analogy of the present study with charged particles confined to a parabolic electric potential inside the hole of a metallic material is straightforward, where the normal modes may be related with the oscillations of the particles around their stable positions.

It should be stressed that if the condition $\Lambda \gg R$ is relaxed, it is possible that some of our results might change. In the case of arbitrary $\Lambda$ the energy of the system may be calculated numerically by using the method presented in Ref. 25 . However the simplicity of the analytical formulation presented here allows us to calculate with high precision the vortex-vortex interaction and the second derivative of the energy.

This paper is organized as follows: In Sec. II we describe the theoretical formalism, while in Sec. III we summarize the numerical approach and briefly mention the stable states obtained from it (see also Ref. 18). Elastic modes for stable vortex configurations are presented in Sec. IV. In Sec. V we relate the softening of elastic modes with the vortex expulsion. A comparison of the elastic mode spectra between highly and nonhighly symmetric configurations is reported in Sec. VI. Section VII deals with the shear and compression content of the elastic modes. We draw our conclusion in Sec. VIII.

\section{THEORETICAL FORMALISM AND NUMERICAL PROCEDURE}

Here we considered a thin disk of radius $R$ and thickness $d$, in which $\Lambda=\lambda^{2} / d \gg R \gg \xi \gg d$, in the presence of a uniform perpendicular magnetic field $\boldsymbol{H}_{0}$. The superconductor is surrounded by vacuum. This allows for neglecting demagnetization effects and one may use $\boldsymbol{H} \approx \boldsymbol{H}_{0}$. We also use dimensionless variables, where lengths are measured in units of the coherence length $\xi$, the vector potential in units of $c \hbar / 2 e \xi$, the magnetic field in units of $H_{c 2}=c \hbar / 2 e \xi^{2}=\kappa \sqrt{2} H_{c}$, and the average energy density in units of $H_{c}^{2} / 8 \pi$. The number of vortices (vorticity) in the system will be denoted by $L$. Also, if necessary, we use the notation presented in Ref. 17 to distinguish vortex configurations with the same $L$; e.g., for $L=6$, $(1,5)$ means 1 vortex in the center with 5 vortices around it, and (6) represents 6 vortices with none of them in the center of the disk.

In a thin disk with constant thickness, as demagnetization effects can be neglected, the vector potential is given by $\boldsymbol{A}$ $=\boldsymbol{A}_{0}=\frac{1}{2} H_{0} \rho \hat{\phi}$. Also we study the system within the London limit, which means that $H \ll H_{c 2}$. In such a limit, the energy of the vortex system is given by ${ }^{14,18}$

$$
\mathcal{G}=\sum_{i=1}^{L}\left(\epsilon_{i}^{\text {self }}+\epsilon_{i}^{\text {shield }}+\sum_{j=1}^{i-1} \epsilon_{i j}\right)+\epsilon^{\text {core }}+\epsilon^{\text {field }},
$$

where

$$
\epsilon_{i}^{\text {self }}=\left(\frac{2}{R}\right)^{2} \ln \left(1-r_{i}^{2}\right)
$$

is the interaction energy between the $i$ th vortex and the boundary of the superconductor (or, in other words, the interaction with its own vortex image),

$$
\epsilon_{i}^{\text {shield }}=-2 H_{0}\left(1-r_{i}^{2}\right)
$$

represents the interaction between the $i$ th vortex and the shielding currents, and

$$
\boldsymbol{\epsilon}_{i j}=\left(\frac{2}{R}\right)^{2} \ln \left[\frac{\left(r_{i} r_{j}\right)^{2}-2 \boldsymbol{r}_{i} \cdot \boldsymbol{r}_{j}+1}{r_{i}^{2}-2 \boldsymbol{r}_{i} \cdot \boldsymbol{r}_{j}+r_{j}^{2}}\right]
$$

is the repulsive energy between vortices $i$ and $j$ (where the interaction with the vortex images was taken into account). Finally, $\epsilon^{\text {core }}=(2 / R)^{2} L \ln (R / a)$ and $\epsilon^{\text {field }}=R^{2} H_{0}^{2} / 4$ are the energies associated with the vortex cores and the external magnetic field, respectively. In the above expressions we use $r_{i}$ $=\rho_{i} / R$ to simplify the notation. Also we made the usual cutoff-i.e., $i=j \rightarrow\left|\rho_{i}-\rho_{j}\right|=a \xi$ (in not normalized units) and $a$ is a constant - to remove the divergence in the self energy terms. As recently shown by us, ${ }^{18}$ the choice $a=\sqrt{2}$ makes the London energy agree well with the Ginzburg-Landau (GL) energy if a contribution $e^{\prime} \approx-1+3 L / R^{2}$ is added to the London energy. However, $\epsilon^{\text {core }}$ and $\epsilon^{\text {field }}$ are only necessary in order to determine the actual state with lowest energy. Since these terms do not depend on the vortex positions (in the London approach), they do not contribute to the results discussed in the present work.

From $\mathcal{G}$ one obtains the force acting on each vortex, by using $-\nabla_{k} \mathcal{G}\left(\boldsymbol{\rho}_{i}, \boldsymbol{\rho}_{j}\right)$, where $-\nabla_{k}$ is the gradient with respect to the coordinate $\boldsymbol{\rho}_{k}$. This yields a force per unit length,

$$
\boldsymbol{F}_{i}=\boldsymbol{F}_{i}^{s}+\sum_{k} \boldsymbol{F}_{i, k}^{i n t}
$$

in units of $H_{c}^{2} \xi / 8 \pi$, where the summation runs over $k$ from 1 to $L$, except for $k=i$. The first term describes the vortex interaction with the current induced by the external field and with the interface,

$$
\boldsymbol{F}_{i}^{s}=\left(\frac{2}{R}\right)^{3}\left(\frac{1}{1-r_{i}^{2}}-\frac{H_{0} R^{2}}{2}\right) \boldsymbol{r}_{i} .
$$

The second term is the vortex-vortex interaction

$$
\boldsymbol{F}_{i, k}^{i n t}=\left(\frac{2}{R}\right)^{3}\left(\frac{\boldsymbol{r}_{i}-\boldsymbol{r}_{k}}{\left|\boldsymbol{r}_{i}-\boldsymbol{r}_{k}\right|^{2}}-r_{k}^{2} \frac{r_{k}^{2} \boldsymbol{r}_{i}-\boldsymbol{r}_{k}}{\left|r_{k}^{2} \boldsymbol{r}_{i}-\boldsymbol{r}_{k}\right|^{2}}\right) .
$$

The above equations allow us to treat the vortices as particles and resemble the energy of a two-dimensional system composed of charged particles with pairwise logarithmic repulsion, confined to some potential. ${ }^{26,27}$ More precisely, the above description is the same as if such charged particles were inside a cylindrical hole surrounded by a metallic material. The shape of such potential is similar to a parabolic potential well for vortices close to the center, but the degree of resemblance to a pure parabolic potential depends on the applied magnetic field for a given number of vortices in the disk (cf. Fig. 1 for the confinement potential for different values of the magnetic field).

\section{NUMERICAL PROCEDURE AND STABLE STATES}

To investigate the elastic modes of the different vortex configurations, we first have to find the stable configurations. 


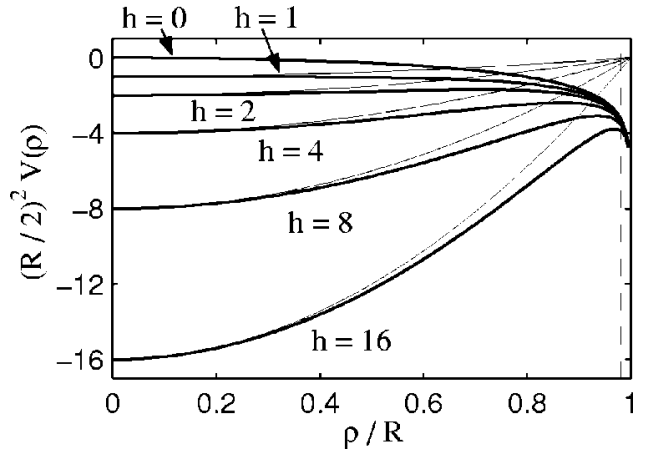

FIG. 1. Confinement potential $\left(V=\epsilon_{i}^{\text {self }}+\epsilon_{i}^{\text {shield }}\right)-$ excluding the vortex-vortex interaction - as a function of the radius $r=\rho / R$, for several magnetic fields $h=R^{2} H_{0} / 2$. This generates the so-called Bean-Livingston barrier (Ref. 28). The vertical dashed line indicates the radial position $r=1-\xi / R$ (for $R=50 \xi$ ), beyond which the London approach breaks down. A pure parabolic confined is represented by the thin lines.

This was accomplished by applying a procedure similar to the one described in Ref. 19 to investigate (meta)stable state close to the equilibrium. First $L^{\prime}$ vortices were distributed randomly inside the disk. Then, we applied a Monte Carlo (MC) simulation with Metropolis algorithm to make the system wander in the configurational space and arrive at the neighborhood of some minimum of $\mathcal{G}_{L}$. After typically $10^{4}$ MC steps, we perform a molecular dynamics (MD) simulation starting from the obtained MC configuration. The final state is achieved after about $10^{6} \mathrm{MD}$ steps. In order to find the ground state (or states with energies very close to it) this trial procedure was repeated several times (more than 1000 times for systems with $L>100$ ), each starting with a different random distribution of $L^{\prime}$ vortices at a given magnetic field $H_{0}$.

The MD simulation was performed by using the BardeenStephen equation of motion ${ }^{29}$

$$
\eta \frac{d \rho_{i}}{d t}=\boldsymbol{F}_{i}
$$

where $i$ represents the $i$ th vortex, $\eta$ is the viscous drag coefficient $\eta \sim \Phi_{0} H_{c 2} / \rho_{n} c^{2}$, with $\rho_{n}$ being the normal-state resistivity. The time integration was accomplished by using the Euler method, but using sufficiently small time steps $\delta t$ in order to assure small vortex displacements between two consecutive steps. The dynamical matrix [cf. Eqs. (1) and (8a)$(8 c)$, whose elements are given by

$$
\frac{\partial^{2} \mathcal{G}_{L}}{\partial \rho_{\alpha, i} \partial \rho_{\beta, j}},
$$

was calculated for the final vortex configuration. In this equation, the Greek indices stand for the components of the vector $\rho_{i}$, while the Italic indices are the labels for the vortices. The computation of the dynamical matrix eigenvalues allowed us to tell whether the given state was stable or unstable (for a stable state all the dynamical matrix eigenvalues must be non-negative). Unstable states were discarded.
Notice that both $\mathcal{G}$ and the forces acting on the vortices diverge at the disk edge, which arises due to the failure of the London limit at $\rho>R-\xi$. To avoid numerical difficulties during the MD simulation, a vortex leaves the disk whenever it was at a distance less than $\xi$ from the disk edge.

For $L=2-9$, vortices may arrange themselves as regular polygons with the possibility that a single vortex stays in the center of the disk ${ }^{14,17,18}$ (of course, for $L=1$, the vortex is in the center of the disk, and no minimization procedure is needed). In these cases, the position of the vortex ring may be found by solving the following equation:

$$
\frac{1}{1-r^{2}}-h+\frac{N \pm 1}{2 r^{2}}-\sum_{n=1}^{N-1} \frac{r^{2}-\cos \phi_{n}}{1+r^{4}-2 r^{2} \cos \phi_{n}}=0
$$

where $N$ is the number of vortices on the ring (or the number of sides of the polygon), $r=\rho_{\text {ring }} / R, \phi_{n}=2 \pi n / N, h=H_{0} R^{2} / 2$, and the plus (minus) sign should be taken if there is one (zero) vortex in the center of the disk. This equation comes from the balance of forces on each vortex.

In addition to the above minimization routines, we also performed Langevin dynamics (LD) simulations, where a thermal fluctuation force $\boldsymbol{\Gamma}_{i}$ is added to Eq. (4). ${ }^{30,31}$ We employed a thermal contribution with zero mean and obeying the fluctuation dissipation theorem

$$
\left\langle\Gamma_{\alpha, i}(t) \Gamma_{\beta, j}\left(t^{\prime}\right)\right\rangle=2 \eta \delta_{\alpha \beta} \delta_{i j} \delta\left(t-t^{\prime}\right) k_{B} T,
$$

where $\langle\cdots\rangle$ means average value, $k_{B}$ is the Boltzman's constant, $T$ is the temperature, and Greek and Italic indices refer to vector components and vortex labels as before. The simulations gave us insight into the time-dependent dynamics of the system, particularly for studying the vortex expulsion. For this purpose we started with a stable configuration at a given magnetic field and decrease the magnetic field by a step $\Delta h=0.05$ (it could be less in certain cases). For each new magnetic field, we started with a small enough temperature (typically $T=10^{-8}$ in units of $H_{c}^{2} \xi^{3} / 8 \pi k_{B}$ ), but sufficient to free the configuration out of a local minimum. The system was cooled down at a low exponential sweep rate [approximately $T(t)=\exp (-t) T_{0}$, where $T_{0}$ was the initial temperature]. We also chose a maximum time step of $\Delta t=10^{-6}$ (in units of $8 \pi \eta / H_{c}^{2}$ ). When the maximum displacement was smaller than $10^{-16}$ or the time at that value of $h$ was greater than 200, we saved the configuration and moved to the next magnetic field. Again, the criterion for vortex expulsion was that it leaves the disk whenever its distance from the edge is smaller than $\xi$ (for computational purposes we considered $R=50 \xi)$.

In Fig. 2 we depict the radius of the vortex ring for the $L=1, \ldots, 9$ states as function of $h=R^{2} H_{0} / 2$ obtained by solving Eq. (6). These states are realized in the limit $R \gg 1$, where the London limit and the GL theory are in good agreement. ${ }^{32}$ The dashed lines represent the radial position after which a net force directed radially outwards acts on the vortex ring. These results for $L>2$ were obtained numerically, while an analytical solution is presented in the Appendix for the case $L=2$. Also the radius of the vortex ring in the absence of images (a system equivalent to classical charged particles in a parabolic confinement) is given by the dotted lines. In this 


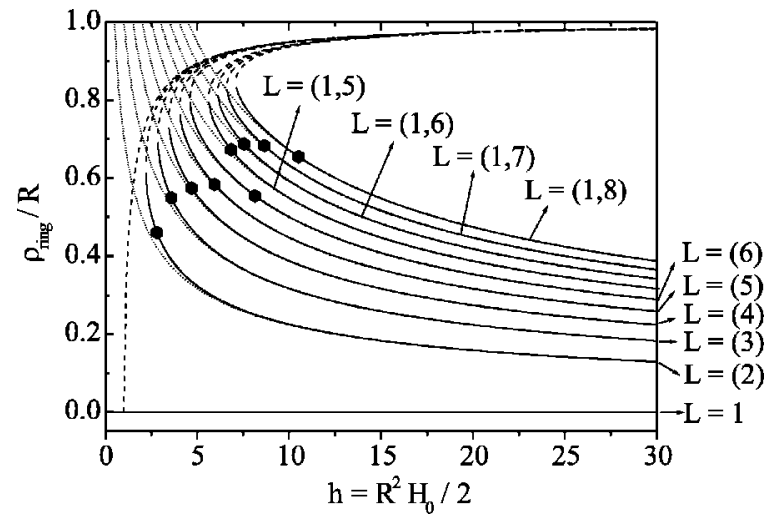

FIG. 2. Radius the vortex ring, $\rho_{\text {ring }}$, in units of $R$ (solid lines), as a function of the magnetic field. The position of the vortex ring in the absence of vortex images is shown by the dotted lines. The dashed lines mark the radial position after which the "entire" vortex regular polygon would be expelled from the disk. The solid dots mark the stability region of the vortex configuration.

case, $\rho_{\text {ring }}$ obeys a simple expression, given by Eq. (A7). One can see that the actual $\rho_{\text {ring }}$ (within the London limit) may be approximated by such simple expression for magnetic fields well above the field in which the configuration becomes stable. Nevertheless, the influence of the images (i.e., the edge of the disk) is important for lower magnetic fields. The ground-state configurations are $L=1,(2),(3),(4),(5),(1,5)$, $(1,6),(1,7)$, and $(1,8)$, although $L=(6)$ and $L=(1,5)$ have very close energies. ${ }^{18}$ In this figure the black dots also show the magnetic fields below which a vortex is pushed away from the disk according to our LD simulations. Notice that vortices are forced to leave the disk at magnetic fields where the regular polygon configuration is still a solution of Eq. (6). The reason for this feature lies in the fact that Eq. (6) is obtained from the radial forces acting on each vortex in a very symmetric configuration. Therefore, the $\rho_{\text {ring }}(H)$ found for each configuration is stable against the radial motion of the entire vortex ring. This does not overrule the possibility that one (or more) vortex moves radially (while the rest of them may move in other directions) and leaves the system.

\section{ELASTIC MODES}

Within the London limit vortices are considered as particles, and the elastic modes of the vortex configurations are obtained by using the dynamical matrix computed for each stable configuration [cf. Eq. (5)]. From Eq. (5), one finds the following expression for the dynamical matrix:

$$
M_{\mathrm{dyn}}=\frac{1}{2}\left(\frac{2}{R}\right)^{4}\left(\begin{array}{cc}
D^{x} & 2 C \\
2 C & D^{y}
\end{array}\right),
$$

with matrix elements

$$
\begin{aligned}
D_{l m}^{x}= & \delta_{l m}\left\{\frac{H_{0} R^{2}}{2}-\frac{1}{1-r_{l}^{2}}\left(1+\frac{2 u_{l}^{2}}{1-r_{l}^{2}}\right)+\sum_{i \neq l}\left[\frac{1}{a_{i l}^{2}}\left(r_{i}^{2}-\frac{2 b_{l i}^{2}}{a_{i l}^{2}}\right)\right.\right. \\
& \left.\left.-\frac{1}{r_{i l}^{2}}\left(1-\frac{2 u_{l i}^{2}}{r_{i l}^{2}}\right)\right]\right\}+\frac{1-\delta_{l m}}{r_{l m}^{2}}\left(1-\frac{2 u_{l m}^{2}}{r_{l m}^{2}}\right) \\
& -\frac{1-\delta_{l m}}{a_{l m}^{2}}\left(1-2 u_{l} u_{m}+\frac{2 b_{l m} b_{m l}}{a_{l m}^{2}}\right),
\end{aligned}
$$

$$
\begin{aligned}
C_{l m}= & -\delta_{l m}\left[\frac{u_{l} v_{m}}{\left(1-r_{m}^{2}\right)^{2}}+\sum_{i \neq l}\left(\frac{b_{l i} c_{l i}}{a_{i l}^{4}}-\frac{u_{l i} v_{l i}}{r_{i l}^{4}}\right)\right]+\left(1-\delta_{l m}\right) \\
& \times\left[\frac{1}{a_{l m}^{2}}\left(u_{l} v_{m}-\frac{b_{l m} c_{m l}}{a_{l m}^{2}}\right)-\frac{u_{l m} v_{l m}}{r_{l m}^{4}}\right]
\end{aligned}
$$

where $\delta_{l m}$ is the Kronecker delta, $r_{i l}=\left|\mathbf{r}_{i}-\mathbf{r}_{l}\right|, a_{i l}^{2}=1-2 \mathbf{r}_{i} \cdot \mathbf{r}_{l}$ $+r_{i}^{2} r_{l}^{2}, \quad u_{i l}=u_{i}-u_{l}, \quad v_{i l}=v_{i}-v_{l}, \quad b_{i l}=r_{l}^{2} u_{i}-u_{l}, \quad c_{i l}=r_{l}^{2} v_{i}-v_{l}, \quad u_{l}$ $=x_{l} / R, v_{l}=y_{l} / R, \mathbf{r}_{l}=\boldsymbol{\rho}_{l} / R$, and the matrix elements $D_{l m}^{y}$ are the same as $D_{l m}^{x}$, but with $v_{i}$ replaced by $u_{i}$. The dynamical matrix is in units of $H_{c}^{2} / 8 \pi \xi^{2}$.

The eigenvalues of the dynamical matrix, Eq. (8a), are analogs to the effective elastic constant $K_{i}$ of the modes given by the eigenvectors of this matrix. Notice that for a nondissipative system ${ }^{19} K_{i}$ is proportional to the eigenmode frequency squared. In this case, a negative value for $K_{i}$ corresponds to an imaginary frequency for the eigenmode, which implies that the system is unstable. For a dissipative system this is also related to the time $\left(\tau_{i}\right)$ required for the exponential overdamped decay of each mode $\left[\exp \left(-t / \tau_{i}\right)\right]$. In the following, we use the related quantity $e_{i}=R^{2} K_{i} / 8 H_{0}$ $=(R / 2)^{4} K_{i} / h$, where $e_{i}$ is of order unity for the highest mode of every $L$ state we found.

For a given $L$ configuration there are $2 L$ degrees of freedom and consequently $2 L$ elastic modes. In highly symmetric configurations - for example, some of the regular polygon configurations with vorticity up to $L=9$ or the $L$ $=(3,9)$ state - degeneracy of some elastic modes is observed.

The modes for $L=4$ at $h=10$ are presented in Fig. $3 .^{33}$ One notices that the lowest mode (with effective elastic constant equal zero) is a pure rotation. The second lowest is the compression of two vortices to the center of the disk while the other two are pushed away. The third and fourth elastic modes are degenerate and represent a translation of the vortex configuration - i.e., is the center-of-mass motion. Degeneracy also happens for the fifth and sixth modes, in which two vortices translate to one side, while the others move in the opposite direction. In the seventh mode vortices form pairs approaching toward each other and at the same time moving farther from the other. The highest mode corresponds to a pure compression; i.e., it is the breathing mode. The elastic modes for $L=19$ at $h=27$ (which corresponds to $H_{0}=0.0216$ if $R=50$ ) also have similar features as those discussed previously. Such a state has a highly symmetric configuration, with 12(6) vortices in the outer (intermediate) ring and 1 vortex in the center. We found the following degeneracy in the normal modes: $\left(e_{3}, e_{4}\right),\left(e_{5}, e_{6}\right),\left(e_{7}, e_{8}\right)$, $\left(e_{10}, e_{11}\right),\left(e_{13}, e_{14}\right),\left(e_{16}, e_{17}\right),\left(e_{18}, e_{19}\right),\left(e_{22}, e_{23}\right),\left(e_{24}, e_{25}\right)$, $\left(e_{27}, e_{28}\right),\left(e_{31}, e_{32}\right),\left(e_{33}, e_{34}\right)$, and $\left(e_{35}, e_{36}\right)$. Some of the modes are depicted in Fig. 4. Again the lowest and highest modes are pure rotation and compression, respectively. Intermediate modes are related to several types of motion, such as inner- and outer-shell opposite rotations $\left(e_{2}\right)$ or opposite compressions $\left(e_{37}\right)$, convectivelike modes (cf. $e_{4}$ and $e_{9}$ ), and mixed rotations and compressions $\left(e_{16}, e_{26}\right.$, and $\left.e_{30}\right)$. These results are similar to the ones previously reported in classical systems of particles, with logarithmic or Coulomb interac- 

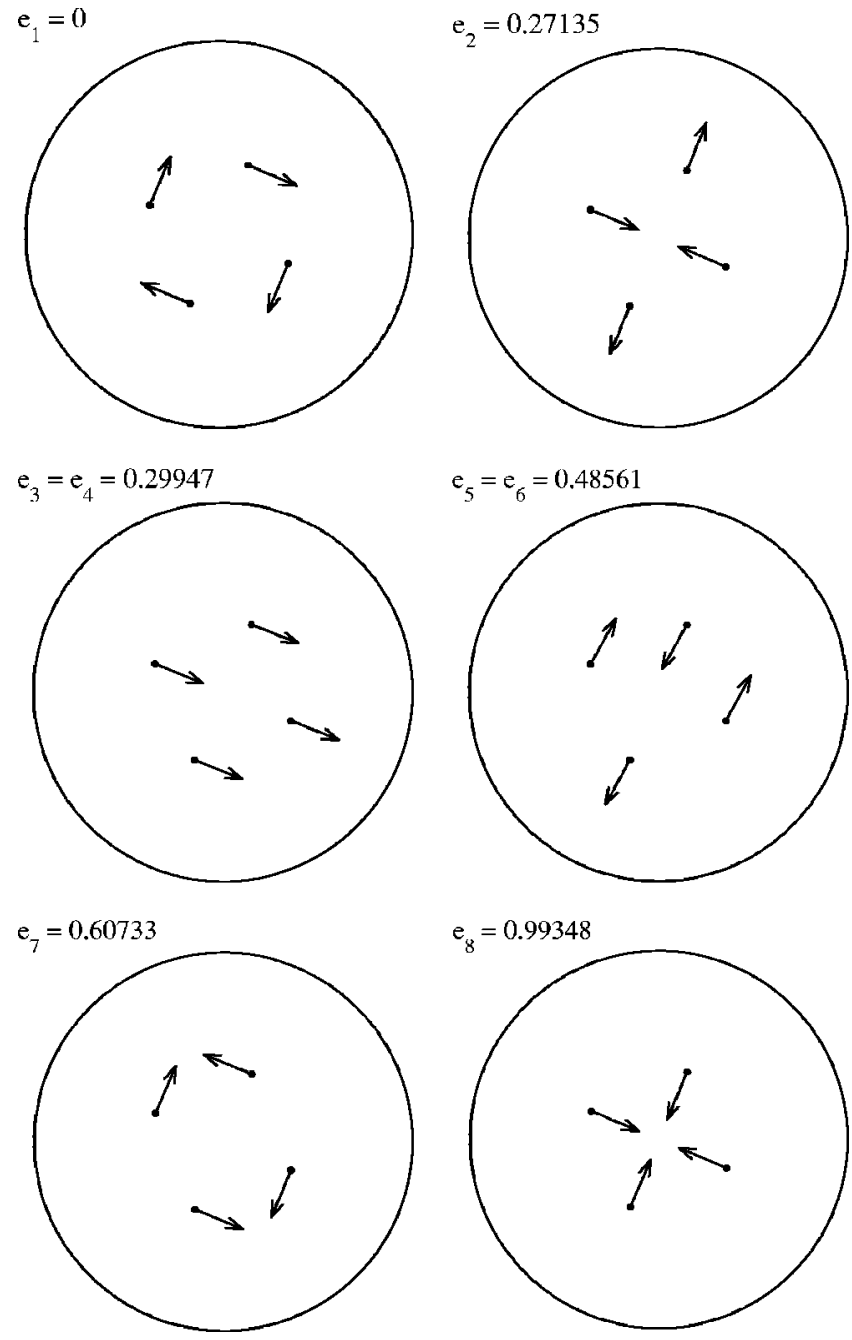

FIG. 3. Elastic modes for $L=4$ at $h=10$. In each frame $e_{i}$ $=(R / 2)^{4} K_{i} / h$, where $K_{i}$ is the effective elastic constant of the mode.

tion, confined to a parabolic potential. ${ }^{19,21}$ This may be explained by the fact that the confining potential in the present case is a mixture of parabolic confinement close to the center of the disk and some sort of deconfining potential [the term $\epsilon_{i}^{\text {self }}$ in Eq. (2a); cf. Fig. 1]. The resemblance to a parabolic potential depends on the magnetic field and on the number of vortices inside the disk.

\section{SOFTENING OF MODES AND VORTEX EXPULSION}

Unlike the classical particle system, which is unbounded, the superconducting disk is bounded by the edge at $R$. Besides, vortices cannot sit inside a sheath close to $R$, whose thickness depends on the magnetic field, as well as on the vortex configuration. This leads to a different dependence of the elastic modes on the magnetic field compared to the one expected if the system were even unbounded, as well as bounded, but with no interactions with the disk edge. To illustrate such difference, one may consider the case $L=2$, which can be solved analytically (see the Appendix). When there is no interaction with the edge, one finds $e_{1}=0$ (rotation of the system as a hole), $e_{2}=e_{3}=1 / 2$ (pure translation of the system in two orthogonal directions), and $e_{4}=1$ (pure compression); each of these values is independent of the magnetic field. In the present case, the confinement (as well as the vortex interactions) is not so simple. As a consequence, although the elastic modes remain the same as the ones obtained for pure parabolic confinement, the degeneracy of the two translation modes is broken and, also, $e_{k}$ increases with increasing $H_{0}$ (except the pure rotation for which $e_{1}=0$ ). These are depicted in Fig. 5, by using the analytical results obtained in the Appendix. As one can see, the elastic modes are less stiffer than the corresponding ones for pure parabolic confinement, but tend to this case when $H_{0}$ is large enough. The magnetic field dependence of $e_{k}$ is not the same for the different modes; for example, the $k=4$ mode has the steepest increase. Also, the translation along the axis joining the two vortices is less stiff than the translation perpendicular to such axis. The translation parallel to the axis joining the two vortices also presents a peculiar feature: while the other modes are positive for $h>\sqrt{3(2 \sqrt{3}+3)} / 2 \approx 2.2018$ [which is also the range over which Eq. (6) admits real solutions; see the Appendix], this mode is negative in the region $\sqrt{3(2 \sqrt{3}+3)} / 2<h<2.7875\left(0.00176 \leqq H_{0} \lesssim 0.00223\right.$ for $R$ $=50$ ), which simply means that $L=2$ is unstable in this region. Hence, the criterion for stability yielded from the elastic modes of a given configuration is more stringent than only finding the solution to Eq. (6). This arises because, in Eq. (6), only the radial forces acting on each vortex in the regular polygon configuration were considered, due to the symmetry of the system. But this neglects the possibility that the solution of Eq. (6) is an unstable solution with respect to some fluctuation in the vortex positions. Therefore, in the specified magnetic field region for $L=2$, the two-vortex system might be in a stable configuration with respect to pure rotation, pure compression, or, even, to translation perpendicular to the axis joining both vortices, but unstable with respect to translation along the axis joining the vortices. This characterizes a saddle point state. This suggests that, if this state is realized for $\sqrt{3(2 \sqrt{3}+3)} / 2<h \leqq 2.7875$, any fluctuation in the vortices positions (due, for example, to thermal fluctuations) could lead to the expulsion of one of the vortices from the disk.

Interestingly, the configurations we have studied do show negative elastic modes-i.e., become saddle point configurations-below a given magnetic field (which depends on the configuration). In order to relate the saddle point states with vortex expulsion, ${ }^{34}$ we analyzed the results obtained from our LD simulations. At each given magnetic field, we also measured the relaxation time (in units of $8 \pi \eta / H_{c}^{2}$ ) required for the maximal and the average vortex displacement (in units of $R$ ) to decrease to $10^{-16}$ (notice that the present system is purely dissipative).

For most of the configurations studied, as soon as the magnetic field reaches the value at which one of the eigenmodes is about to become negative, a vortex is expelled from the disk. For example, our results for $L=2$ show that a vortex is expelled at the magnetic field below which the lowest elastic mode becomes negative-i.e., at $h=2.7875$. But for some configurations, as for example the $L=(1,8)$ state, $^{35}$ a change in the configuration geometry may happen first, keep- 

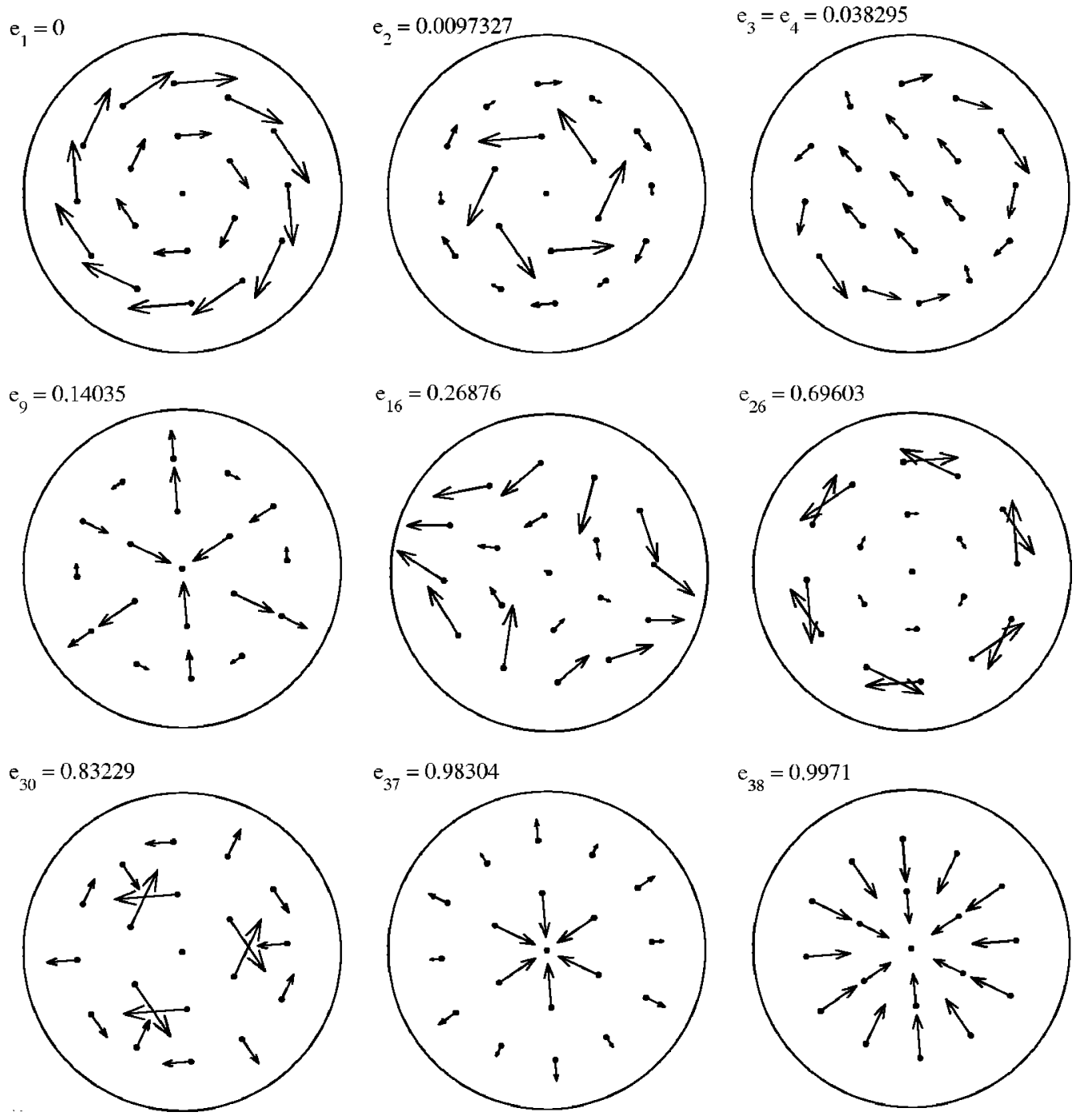

FIG. 4. The same as Fig. 3 but now for $L=19$ at $h=27$.

ing the vorticity fixed, before a vortex is expelled. In this case, at a high enough magnetic field, there are eight vortices in a ring of radius $\rho_{\text {ring }}$ plus a central vortex. At $h=10.85$ (the magnetic field below which this configuration turns to be a saddle point) vortices arrange themselves in a deformed ring

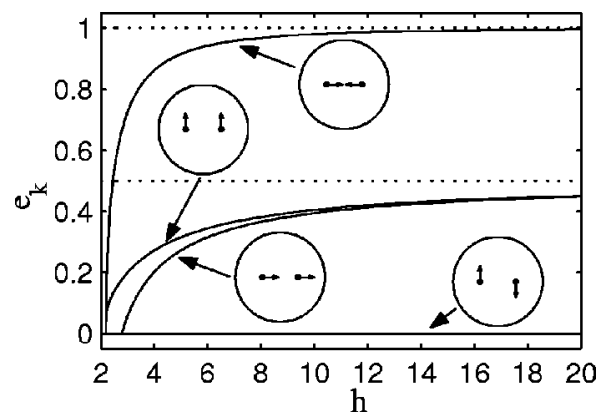

FIG. 5. Dependence of $e_{k}$ upon $h=H_{0} R^{2} / 2$ for $L=2$ (see the Appendix for the analytical expressions). The displacements corresponding to the elastic modes are shown in the insets. The dotted lines represent $e_{k}$ when neglecting the vortex images and including only a pure parabolic potential. or, equivalently, the initial perfect ring splits into two groups of four vortices, each with a distinct radius. To confirm this feature, in addition to the LD simulations we also solved numerically the system of two equations,

$$
\frac{8 r_{i}^{8}}{1-r_{i}^{8}}-2 h r_{i}^{2}+5+8 r_{i}^{4}\left(\frac{1}{r_{i}^{4}+r_{j}^{4}}-\frac{r_{j}^{4}}{1+r_{i}^{4} r_{j}^{4}}\right)=0,
$$

where $i=a, b, r_{a}=\rho_{a} / R$, and $r_{b}=\rho_{b} / R$ are the two different radius of each group of four vortices. This equation is obtained from the radial forces [Eqs. (3a)] acting on vortices at different rings. In Fig. 6(b) the LD results (symbols) and the solutions of Eqs (9) (lines) are presented. Both agree very well. The relaxation time for the maximal and average vortex displacements are also shown. Notice that close to the change in the configuration symmetry and to the vortex expulsion the relaxation time increases steeply. The modes spectrum for the lowest energy are shown in Fig. 6(a). There is a drastic change in the elastic mode spectrum exactly at $h=10.85$. Above this field the second elastic mode consists of the outward and inward motion of alternate vortices. Interestingly, the second mode at $h>10.85$ is represented by 


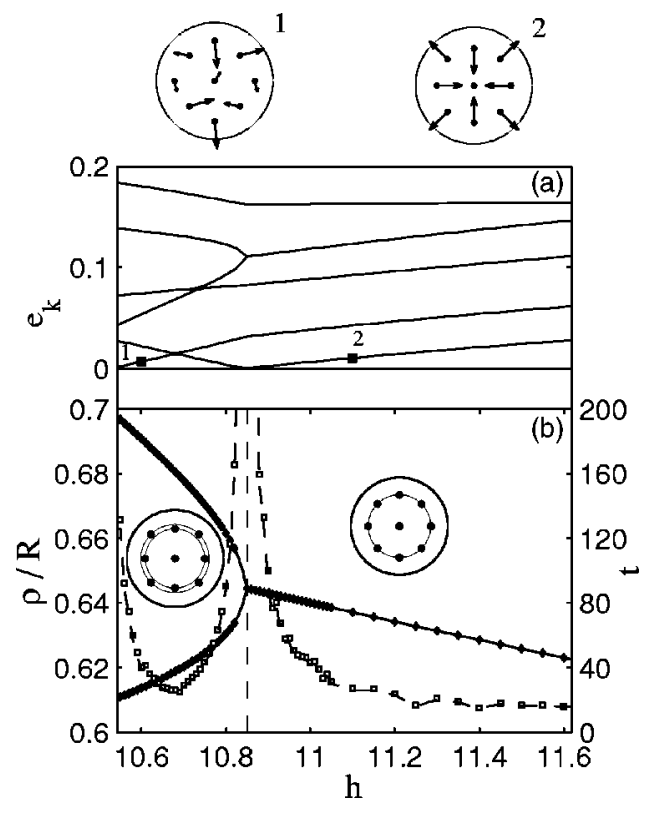

FIG. 6. (a) Dependence of $e_{k}$ upon $h$ for $L=9$. Points 1 and 2 refer to the eigenmodes shown on the top. (b) Possible force-free vortex configurations as function of $h$. The solid lines are the solution of Eq. (9) and the symbols are the results from the MD simulations. The open squares (dashed line) show the relaxation time required for the maximal (average) vortex displacement to decrease to $10^{-16}$. Above $h=10.85$ (marked by the vertical dashed line), the configuration with one vortex in the center and eight vortices on a ring is stable (inset on the right). Below that field, when the second elastic mode is about to become negative, the eight vortices arrange themselves in two rings (inset on the left). For $h<10.54$ the $L=9$ state is no longer stable.

the outward and inward motion of alternate vortices, as indicated by figure with the label 2 on top of Fig. 6 . At $h$ $=10.85$ this mode has $e_{2}=0$. Decreasing $h$ further, with the motion of four of the vortices to an outer radius, this mode becomes stiffer, and eventually the mode indicated by label 1 turns out to be the second lowest mode. It suggests that not all four vortices which are closer to the edge are expelled and, below $h \approx 10.54$, one vortex leaves the system. In fact, our LD results show that small deviations from the solutions of Eq. (9) do occur and such deviations become more pronounced for one given vortex until its ultimate expulsion.

\section{CONFIGURATION SYMMETRY AND MODE SPECTRA}

To illustrate the influence of the configuration symmetry on the modes, we plotted the evolution of $e_{k}$ with the magnetic field for $L=(3,8)$ and $L=(3,9)$ in Fig. 7. As for $L=2$, for both configurations $e_{k}$ increases with $H_{0}$ (except $e_{1}$ which remains zero within the numerical accuracy $10^{-8}$ ), but some modes are more sensitive than the others upon changes in $H_{0}$. This means that the vortex configurations become stiffer with increase of $H_{0}$, because the force responsible for the confinement of the vortices [proportional to $H_{0}$, cf. Eq. (3a)] becomes stronger. Moreover, some modes present a pronounced dependence on the magnetic field, while others are

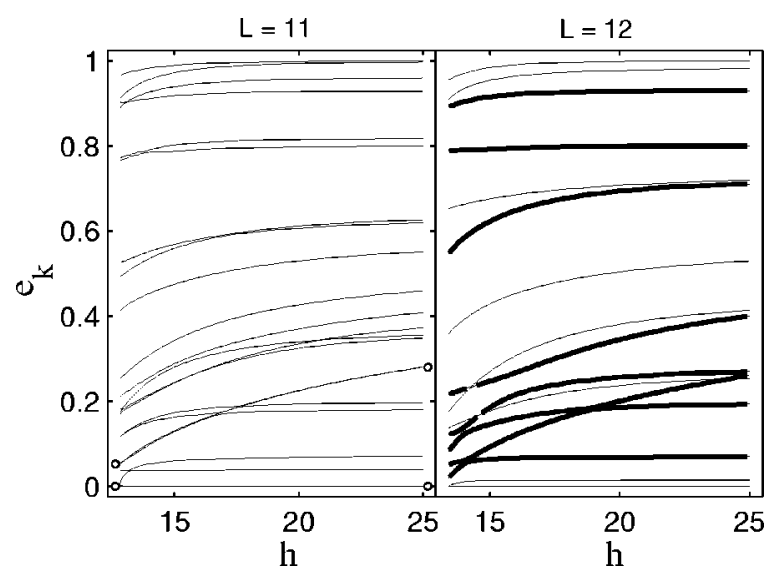

FIG. 7. Dependence of $e_{k}$ upon $h=H_{0} R^{2} / 2$ for $L=(3,8)$ and $L$ $=(3,9)$. Thick lines depict twofold degenerate modes. For $L=11$, circles indicate modes whose difference is too small to be observed, as in the case of the $e_{1}=0$ and $e_{2}$ modes (difference $<10^{-4}$ ).

practically not influenced. Two main features may happen with an increase of the magnetic field. First, a crossover between different types of modes may occur at a given magnetic field. Second, modes at lower fields can be different than those at higher fields. The first feature is observed with the modes related to a translation of the entire configuration and other modes, for example, for $L=12$ (depicted in Fig. 8). At low fields (for example at $h<18$ ), the two degenerate modes associated with translation are the fifth and sixth lowest ones, while for $h>19$ they appear as the seventh and eighth modes. The other two modes depicted in this figure do not present exactly the same vortex motions for $h=15$ and $h=20.1$.

Since the $L=12$ configuration is more symmetric, eight eigenvalues of the dynamic matrix are twofold degenerate (shown by thick lines). On the contrary, $L=11$ present only nondegenerate modes (although the difference between the first and second modes is only about $10^{-5}$ ). Also the second mode for $L=12$ has much larger value (and is clearly nonzero) compared with its respective mode in the $L=11$ configuration. The higher symmetry decreases the number of degrees of freedom of the system, making easy direction motions less likely. As a result the effect of fluctuations (as, for instance, temperature) experienced by the vortex configurations for $L=12$ are likely to be different than for the $L$ $=11$ configuration.

In order to test this conjecture, we obtained the vortex trajectories for $L=12$ and $L=11$ at $h=16$ from our LD simulations. This was performed by computing the vortex positions at $\Delta t=0.1$ time steps between $t=35$ and $t=200$. The results for two different temperatures-namely, $T=0.0001$ and $T=0.001$ - are plotted on the left and right of Fig. 9, respectively. The vortex trajectories are mainly oriented in the azimuthal direction (see Ref. 36 for the equivalent system of charges). Nevertheless, for both temperatures, the azimuthal range of the trajectories is quite different for the vortices in the inner shell for $L=11$ (top) and $L=12$ (bottom). In the former case, the trajectories within the inner shell are of the same size or larger than the ones in the outer shell. Meanwhile, for $L=12$, the trajectories in the different shells seem 

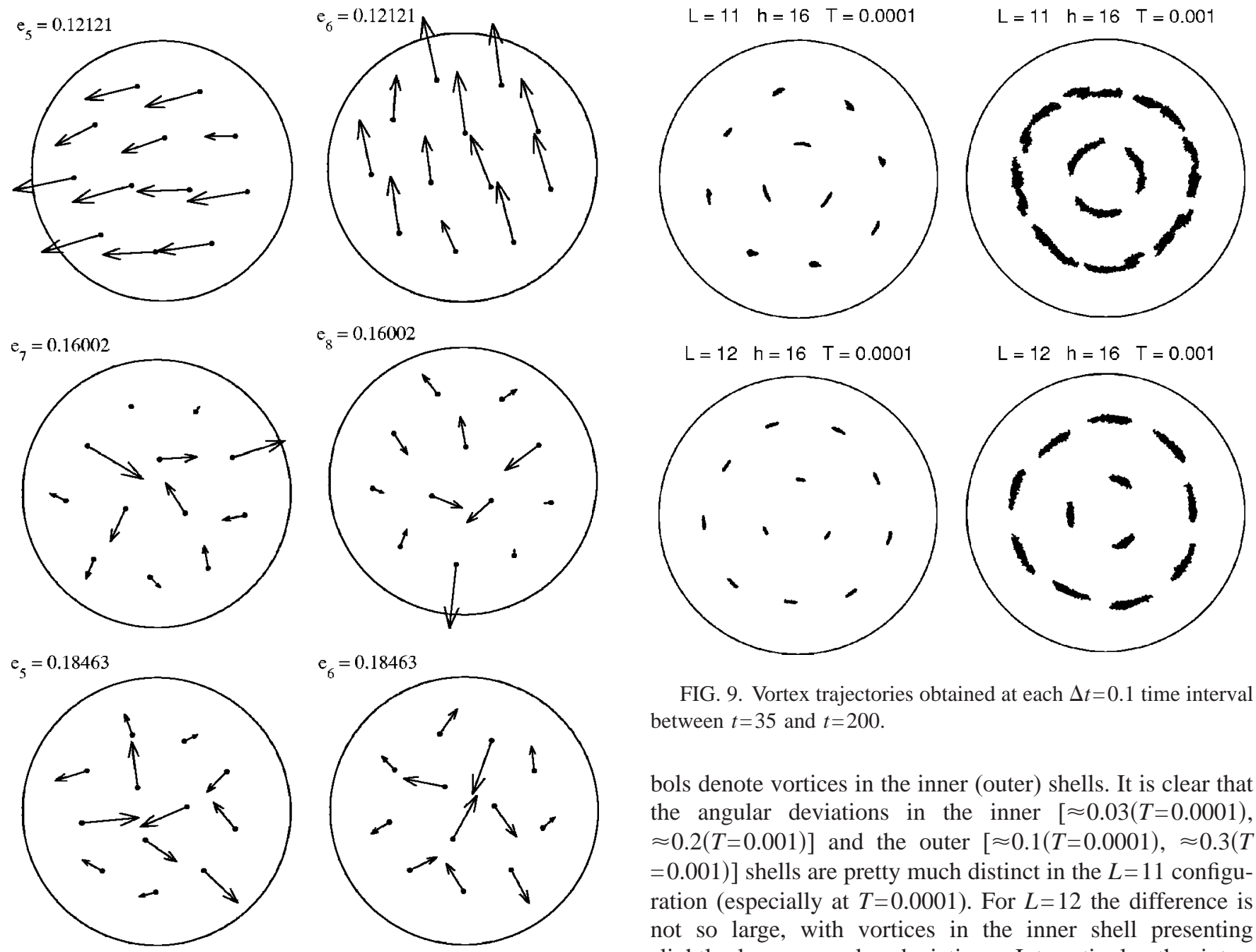

FIG. 9. Vortex trajectories obtained at each $\Delta t=0.1$ time interval between $t=35$ and $t=200$.

bols denote vortices in the inner (outer) shells. It is clear that the angular deviations in the inner $[\approx 0.03(T=0.0001)$, $\approx 0.2(T=0.001)]$ and the outer $[\approx 0.1(T=0.0001), \approx 0.3(T$ $=0.001)]$ shells are pretty much distinct in the $L=11$ configuration (especially at $T=0.0001$ ). For $L=12$ the difference is not so large, with vortices in the inner shell presenting slightly larger angular deviations. Interestingly, the intershell difference in $\Delta \phi$ for $L=11$ decreases at $T=0.001$, which seems to be related with the increase in the deviation in the radial direction (see Fig. 9). A similar result was found for a colloidal system confined in a hard wall potential. ${ }^{37}$ The angular distribution of the vortices was also calculated in order to analyze the correlation between fluctuations in different shells. For this purpose, at each $\Delta t=0.01$ time step, we measured the angular position of the vortices with respect to a given reference vortex in the inner shell and computed a histogram with these values. The results are plotted in Fig. 10(b). Thick (thin) lines represent the outer (inner) shell vortices. The angular distribution in the inner shell - for both configurations and at different temperatures - presents peaks at $2 \pi / 3$ and $4 \pi / 3$, which indicates that intrashell vortex fluctuations are correlated. But a noticeable difference between the $L=11$ and $L=12$ configurations appears in the angular distribution of the outer-shell vortices. For $L=12$ the vortex fluctuations in the outer shell (thick lines) are somewhat locked to the fluctuations in the inner shell, as indicated by the well defined peaks. Meanwhile, for $L=11$, vortex fluctuations in the outer shell are not well correlated with the ones in the inner shell, particularly at $T=0.001$. These features seem to be linked with the fact that the second eigenmode is distinctly nonzero for $L=12$, while it is almost zero for $L=11$. Therefore, for the latter configuration different 


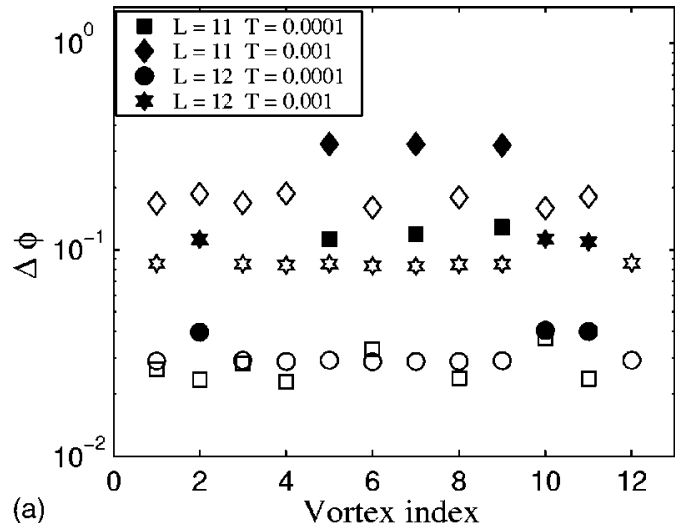

(a)

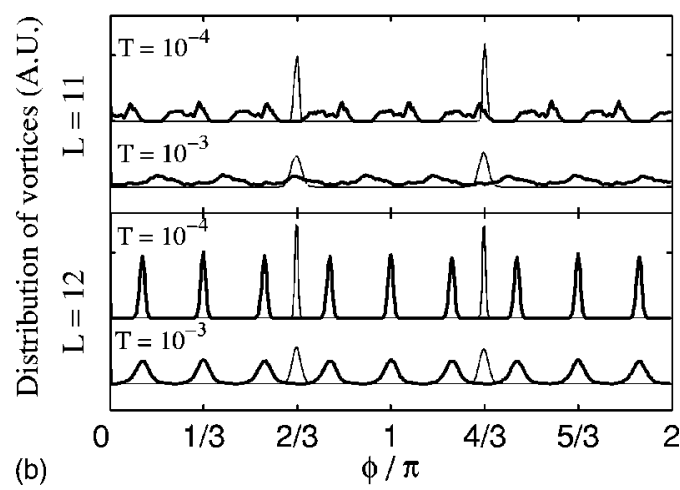

FIG. 10. (a) Vortex angular deviation for $L=11$ and $L=12$ at $h$ $=16$, obtained at two different $T$. The solid (open) symbols represent vortices in the inner (outer) shell. (b) Distribution of vortices obtained at different time intervals as function of their angular positions with respect to a given vortex in the inner shell. Thick (thin) lines represent vortices in the outer (inner) shell.

types of modes other than pure rotation are easily accessible, which is not the case for $L=12$. This shows how the symmetry of the $L=(3,9)$ configuration (especially the clear nonzero value of the second elastic mode) plays a role in the manner vortices move and we expect that similar features can be observed in other highly symmetric configurations.

\section{SHEARLIKE AND COMPRESSIONLIKE MODES}

The elastic modes in infinite thin superconducting films are usually characterized by the compression $\left(C_{11}\right)$ and shear $\left(C_{66}\right)$ moduli. ${ }^{10,11}$ Following Ref. 38, in disks, one may investigate shearlike and compressionlike modes by computing $\nabla \times s_{i, k}$ and $\nabla \cdot s_{i, k}$, where $s_{i, k}$ are the interpolated eigenvector field obtained from the eigenvectors associated with the $i$ th mode. The interpolation was performed in a square grid, and the curl and divergence of the eigenvector field were calculated numerically. ${ }^{38}$ A grid with $N^{2}=120 \times 120$ points was chosen and cubic polynomials were interpolated between the eigenvector values. From the divergence and curl of the eigenvector field, one can also obtain the quantities

$$
f_{c}(i)^{2}=\frac{1}{N^{2}} \sum_{k=1}^{N^{2}}\left|\nabla \times s_{i, k}\right|^{2},
$$
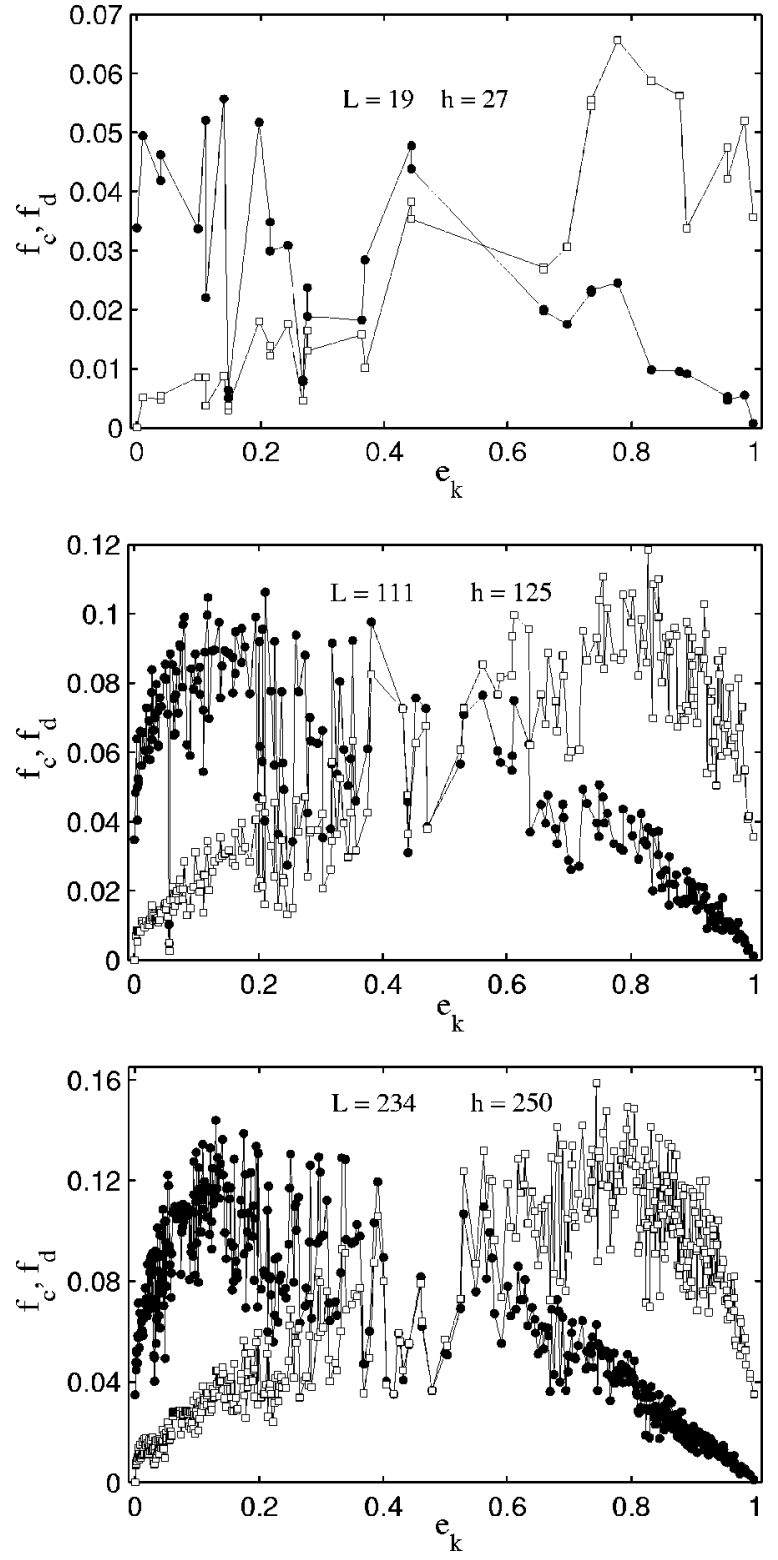

FIG. 11. Shear $f_{c}$ (solid circles) and compression $f_{d}$ (open squares) content of each mode as given by Eq. (10) for the $(1,6,12)$ state at $h=27, L=111$ at $h=125$, and $L=234$ at $h=250$.

$$
f_{d}(i)^{2}=\frac{1}{N^{2}} \sum_{k=1}^{N^{2}}\left|\nabla \cdot s_{i, k}\right|^{2},
$$

which describe how much each mode is shearlike or compression like.

In Fig. 11 we show the calculated $f_{c}$ and $f_{d}$ as function of $e_{k}$ for 19 vortices at $h=27,111$ vortices at $h=125$, and 234 vortices at $h=250$. These are the configurations with lowest energy (for the given magnetic fields and number of vortices) found by our numerical approach and within the usual London limit. ${ }^{18}$ It is clear that the shearlike (compressivelike) modes occur for smaller (larger) $e_{k}$. Similar dependence for $f_{d}$ and $f_{c}$ as function of the eigenfrequencies was observed experimentally for charges interacting via a (screened) Cou- 


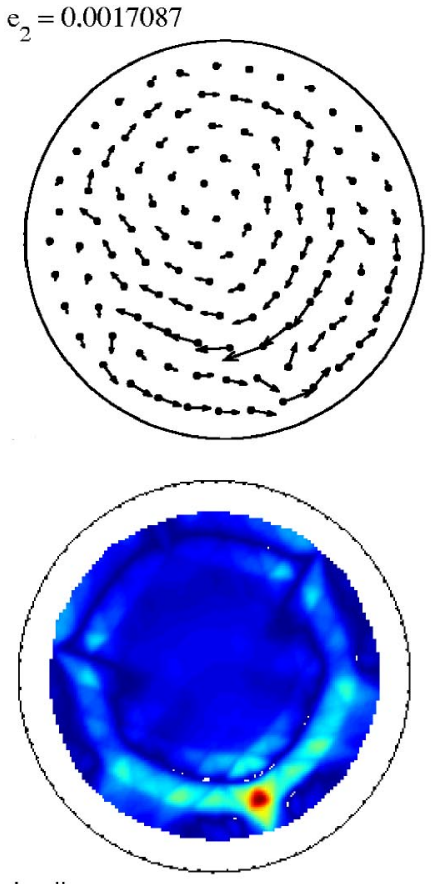

|curl|

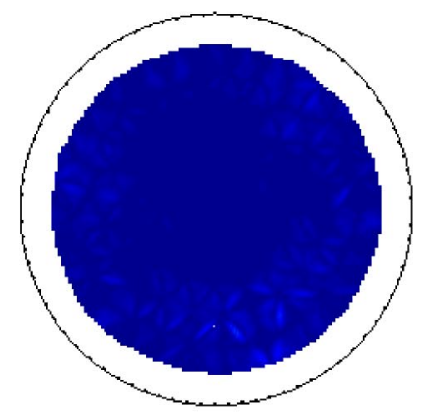

$|\operatorname{div}|$

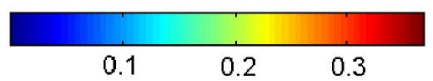

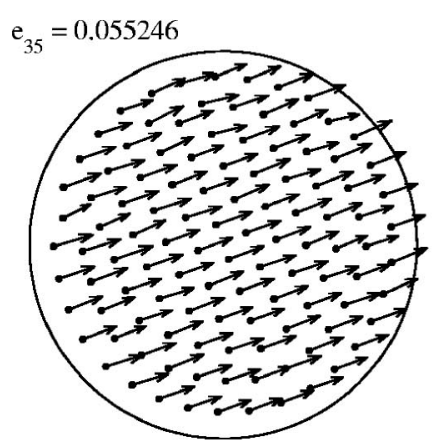
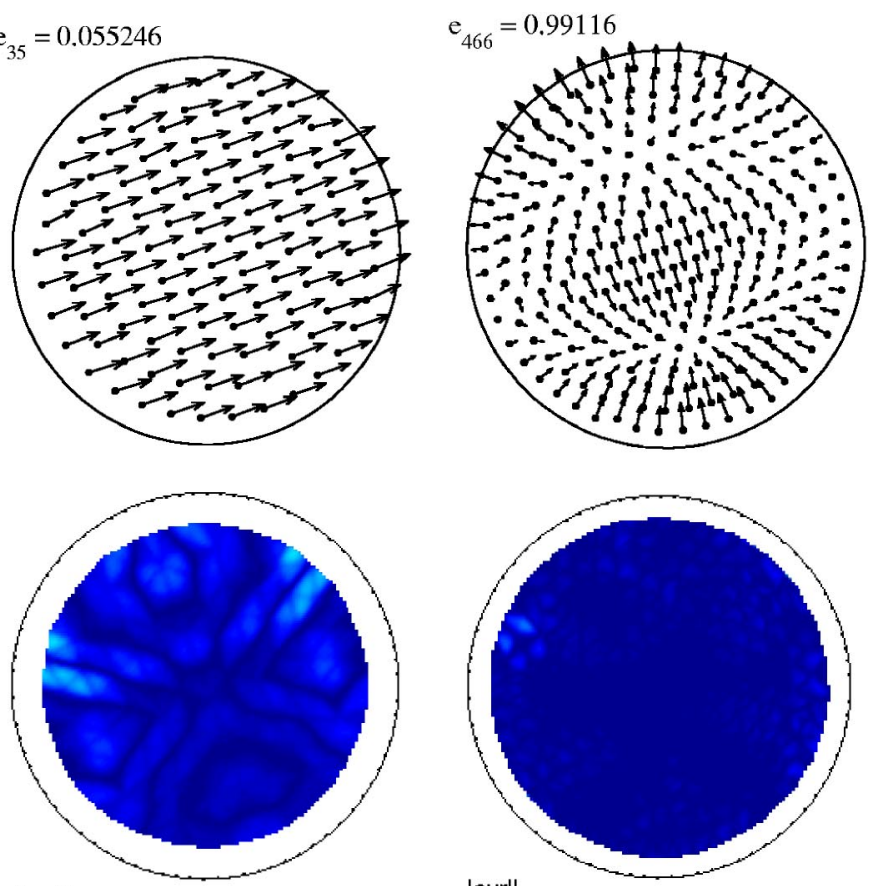

|curl|
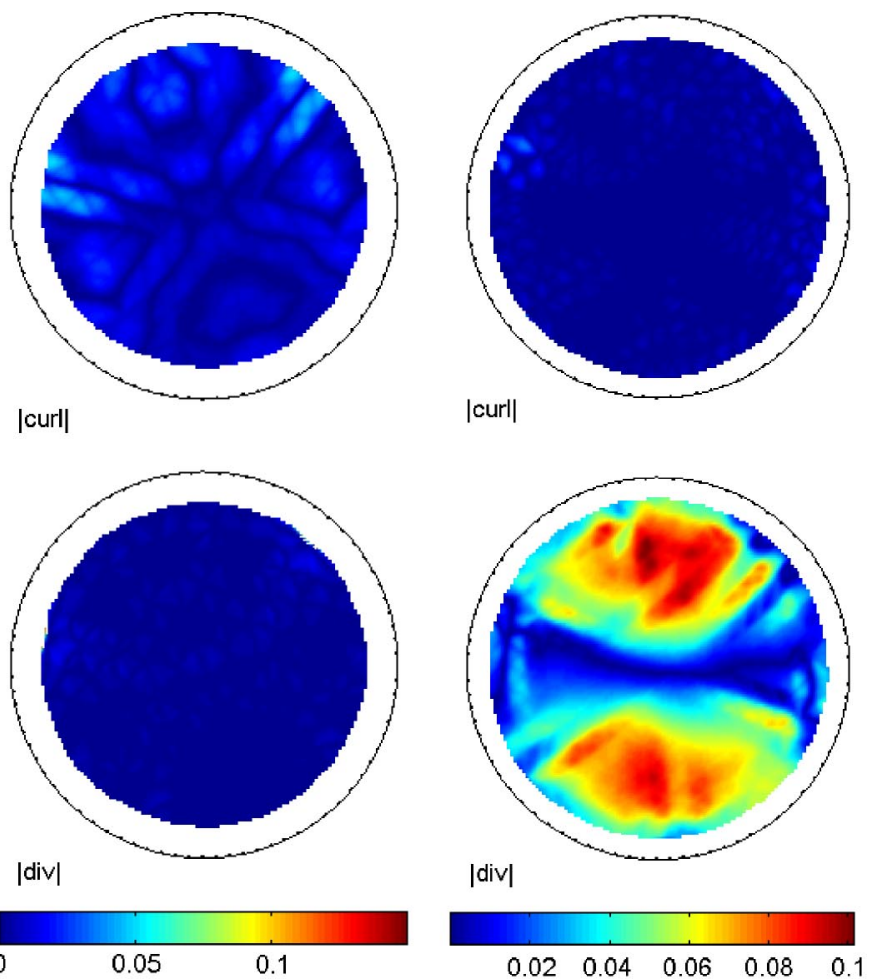

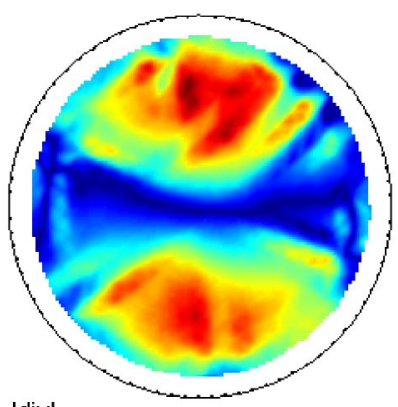

|div|

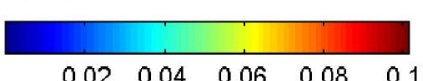

FIG. 12. (Color online) From left to right: modes $k=2$, 35, for $L=111$ at $h=125$, and $k=466$ for $L=234$ at $h=250$. Below each configuration a density plot of the absolute value of the $\nabla \times s_{i, k}$ (curl) and $\nabla \cdot s_{i, k}$ (div) is presented.

lomb interaction and confined to a parabolic potential ${ }^{39}$ (notice that $e_{k}$ plays a role here similar to the eigenfrequencies of the normal modes for particles confined to potential wells). For such a high number of vortices (and at these magnetic fields) $f_{c}$ is maximal around $e_{k} \sim 0.2$, while $f_{d}$ around $e_{k} \sim 0.8$. In addition, the region around $e_{k} \sim 0.5$ exhibits a density of modes with approximate equally shear and compressive content. After (before) this region the shearlike (compressionlike) behavior decays (increases) practically linearly. Other configurations with the same or similar number of vortices at those given magnetic fields exhibit a similar behavior.

In order to give an idea of the curl and the divergence fields obtained from the eigenvectors, Fig. 12 shows the eigenmodes (on top) and their respective spatial dependence of $\left|\nabla \times s_{k}\right|$ and $\left|\nabla \cdot s_{k}\right|$. The $k=2$ mode $(L=111$ and $h=125)$ is a more pronounced shearlike mode, since $\left|\nabla \times s_{k}\right|$ varies from 0 to 0.3 , while $\left|\nabla \cdot s_{k}\right|$ is roughly zero. The opposite happens for $k=466(L=234$ and $h=250)$, making this mode more compressive like. The translation mode is obtained at $k=35$ for 111 vortices and, as expected, does not have a well-defined shearlike or compressivelike behavior, since both $\left|\nabla \times s_{k}\right|$ and $\left|\nabla \cdot s_{k}\right|$ are very small.

Finally, to investigate further the dependence of the elastic modes on the magnetic field, we computed $f_{d}$ and $f_{c}$ for the $L=(3,9)$ configuration as function of $h$ (see Fig. 13). First, a big dip in $f_{d}$ and $f_{c}$ occurs at $e_{k} \sim 0.12$ for $h=16$, but moves to $e_{k} \sim 0.2$ for $h=20.5$. This dip comes from the (twofolddegenerate) modes related with the entire translation of the system, as seen in Fig. 12 for $k=35$ for 111 vortices. As pointed out before (cf. Fig. 8), this change in the dip position is due to the greater stiffness dependence of these modes on $h$. Also, $h=20.5$, shearlike modes are more distinguished from the compressivelike ones for $e_{k}<0.2$, with somewhat equally shearlike and compressivelike modes at $0.2<e_{k}$ $<0.7$. At $h=16$, the transition from shearlike to compressive- 

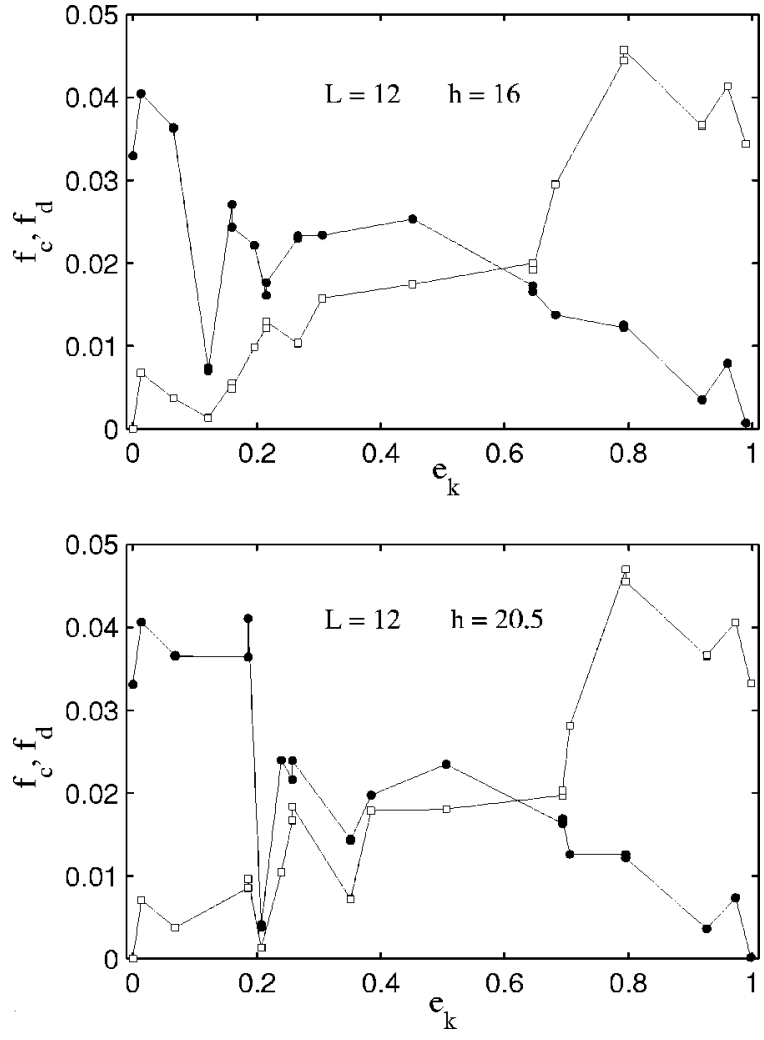

FIG. 13. $f_{c}$ (solid circles) and $f_{d}$ (open squares) for the $L$ $=(3,9)$ state at $h=16$ (top) and $h=20.5$ (bottom).

like modes is less steep. Moreover, at $h=16$, compressivelike modes start appearing when $e_{k}>0.65$, but such a feature occurs at $e_{k} \sim 0.7$ for $h=20.5$.

\section{CONCLUSIONS}

In this paper, we investigated the elastic modes of vortex systems in superconducting thin disks. For such a purpose the dynamical matrix was computed from stable vortex configurations, and its eigenvalues and eigenvectors were found. This study is also relevant for the understanding of the normal modes of charged particles confined to a finite cylindrical metallic hole.

We observed that the stable vortex configurations have a pure rotation of the entire vortex system as the lowest eigenmode. This is in agreement with recent results from simulations of vortices in Corbino disks, ${ }^{40}$ which tells us that the vortex arrangements rotate as a rigid body within the elastic limit. The highest eigenmode is related to the so-called breathing mode-i.e., a compression of the configuration as a whole. For low vorticity values, the second lowest eigenmode usually corresponds to the intershell rotation, while the creation of whirl and movement of vortices takes place at higher vorticity.

The calculated eigenmodes present a marked dependence on the magnetic field. Distinct modes may behave differently as function of the magnetic field. The softening of the modes yields a threshold magnetic field below which some of the computed eigenvalues are negative. This indicates a saddle point configuration, marking a transition to a different stable configuration. Such a feature is also attested by the analytical solution for a two-vortex system.

We also analyzed the shear and compressive contents of the eigenmodes. Shearlike modes are less stiffer and are have mostly eigenvalues below $e_{k}=0.5$. Compressivelike modes are mostly found with $e_{k}>0.5$. The mode corresponding to a translation of the whole vortex system (i.e., the slushing mode) has a relative low eigenvalue.

\section{ACKNOWLEDGMENTS}

This work was supported by the Flemish Science Foundation (FWO-V1), the "Onderzoeksraad van de Universiteit Antwerpen" (GOA), the Belgian Science Policy, the European ESF-Vortex Matter, and the Brazilian Science Agency CAPES. We acknowledge useful discussions with B. Partoens, M. Kong, and G. Papp.

\section{APPENDIX: TWO VORTICES IN THE LONDON LIMIT}

In this appendix we derive analytical expressions for the $L=2$ configuration. More specifically we find the dependence on $H_{0}$ of both the vortex ring radial position and the normal modes.

For $N=2$ Eq. (6) reduces to

$$
2 h r^{6}+3 r^{4}-2 h r^{2}+1=0
$$

with $0<r<1$. Therefore one has to solve a third-degree polynomial in $r^{2}$. The physical solutions $(0<r<1$ and $\operatorname{Im}\{r\}=0)$ arise when

$$
\frac{1}{(2 h)^{4}}+\frac{2}{3} \frac{1}{(2 h)^{2}}-\frac{1}{27}<0
$$

or, equivalently,

$$
H_{0}>\frac{\sqrt{3(2 \sqrt{3}+3)}}{R^{2}}
$$

The three real roots for $r^{2}$ are given by

$$
r_{n}^{2}=2 \sqrt{\frac{1}{3}+\frac{1}{(2 h)^{2}}} \cos \left(\frac{\alpha+2 n \pi}{3}\right)-\frac{1}{(2 h)^{2}},
$$

with $n=0,1,2$, and $\alpha$ is determined by

$$
\cos \alpha=-\frac{1+(2 h)^{2}}{\left[1+\frac{(2 h)^{2}}{3}\right]^{3 / 2}}
$$

Obviously, for $n=2$ we have a purely imaginary solution for $r$. The other two solutions for $r$ represent radial positions in which the vortex polygon is pushed towards the edge, $r_{u}$ $=\rho_{u} / R$, and is stable (concerning motion in the radial direction), $r_{s}=\rho_{s} / R$. Therefore, 


$$
\begin{gathered}
\rho_{u}=H_{0}^{-1 / 2} \sqrt{2 \sqrt{1+\frac{H_{0}^{2} R^{4}}{3}} \cos \frac{\alpha}{3}-1}, \\
\rho_{s}=H_{0}^{-1 / 2} \sqrt{\sqrt{1+\frac{H_{0}^{2} R^{4}}{3}}\left(\sqrt{3} \sin \frac{\alpha}{3}-\cos \frac{\alpha}{3}\right)-1},
\end{gathered}
$$

where $\alpha$ is given by Eq. (A4b) remembering that $2 h=H_{0} R^{2}$. In Fig. 2, for the case $L=2$, solid and dashed lines depict, respectively, $\rho_{s}$ and $\rho_{u}$ as function of $H_{0}$. Expanding Eqs. (A5) for large $H_{0} R^{2}$ (or, equivalently, for small $1 / 2 h$ ) and neglecting terms of order $(1 / 2 h)^{2}$, one obtains

$$
\rho_{u} \approx \sqrt{1-\frac{2}{H_{0} R^{2}}}
$$

and

$$
\rho_{s} \approx H_{0}^{-1 / 2} .
$$

The first expression comes from the balance of the inward shielding and the outward image forces acting on a vortex, neglecting the interaction with other vortices and their images. This should be realized when such vortex is close to the edge. So it is natural that when the magnetic field increases, $\rho_{u}$ moves towards the disk edge with Eq. (A6a) as the limit. The second expression is simply the position of the twovortex ring if there were no images-i.e., no interaction with the disk edge. It is easy to find from Eq. (6), neglecting the terms due to the interactions with images, that

$$
\rho^{*}=\sqrt{\frac{N \pm 1}{H_{0}}},
$$

where the plus (minus) sign should be taken when one (zero) vortex is the center of the regular polygon configuration with $N$ sides. For $L=N=2$, this reduces to Eq. (A6b). Dotted lines representing Eq. (A7) are shown in Fig. 2 for $L=1 \rightarrow 8$. For each of these configurations the actual $\rho_{s}$ has Eq. (A7) as a limit, since vortices are more closely packed when the magnetic pressure increases, thus diminishing the interaction with the disk edge.

We now turn to the problem of finding the elastic modes for $L=2$. If we consider that the vortices sit at $\left(\rho_{s}, 0\right)$ and $\left(-\rho_{s}, 0\right)$, the dynamical matrix takes the following form in this case

$$
M_{\mathrm{dyn}}=\frac{1}{2}\left(\frac{2}{R}\right)^{4}\left(\begin{array}{cccc}
D_{1}^{x} & D_{2}^{x} & 0 & 0 \\
D_{2}^{x} & D_{1}^{x} & 0 & 0 \\
0 & 0 & D_{1}^{y} & D_{2}^{y} \\
0 & 0 & D_{2}^{y} & D_{1}^{y}
\end{array}\right),
$$

where

$$
\begin{gathered}
D_{1}^{x}=\frac{H_{0} R^{2}}{2}-\frac{1}{1-r^{2}}\left(1+\frac{2 r^{2}}{1-r^{2}}\right)+\frac{1}{(2 r)^{2}}-\frac{r^{2}}{\left(1+r^{2}\right)^{2}}, \\
D_{2}^{x}=-\left[\frac{1}{\left(1+r^{2}\right)^{2}}+\frac{1}{\left(2 r^{2}\right)^{2}}\right],
\end{gathered}
$$

$$
\begin{gathered}
D_{1}^{y}=\frac{H_{0} R^{2}}{2}-\frac{1}{1-r^{2}}-\frac{1}{(2 r)^{2}}+\frac{r^{2}}{\left(1+r^{2}\right)^{2}}, \\
D_{2}^{y}=-\left[\frac{1}{\left(1+r^{2}\right)^{2}}-\frac{1}{\left(2 r^{2}\right)^{2}}\right],
\end{gathered}
$$

and $r=\rho_{s} / R$. The eigenvalue problem leads to

$$
\left(\begin{array}{cc}
D_{1}^{i} & D_{2}^{i} \\
D_{2}^{i} & D_{1}^{i}
\end{array}\right)\left(\begin{array}{l}
s_{1 i} \\
s_{2 i}
\end{array}\right)=0
$$

with

$$
\lambda_{ \pm}^{i}=\frac{1}{2}\left(\frac{2}{R}\right)^{4}\left(D_{1}^{i} \pm D_{2}^{i}\right)
$$

Above, $i=x$ or $y, s_{1 i}\left(s_{2 i}\right)$ is the eigenvector components in the $i$ direction for particle 1 (2) and $\lambda_{ \pm}^{i}$ are the eigenvalues. A straightforward calculation yields the following eigenvalues:

$$
\begin{gathered}
\lambda_{-}^{y}=0, \\
\lambda_{+}^{y}=\frac{8}{R^{2}}\left[\frac{H_{0}}{2}-\frac{1}{R^{2}-\rho_{s}^{2}}-\frac{R^{2}-\rho_{s}^{2}}{\left(R^{2}+\rho_{s}^{2}\right)^{2}}\right], \\
\lambda_{+}^{x}=\frac{8}{R^{2}}\left[\frac{H_{0}}{2}-\frac{1}{R^{2}-\rho_{s}^{2}}\left(1+\frac{2 \rho_{s}^{2}}{R^{2}-\rho_{s}^{2}}\right)-\frac{1}{R^{2}+\rho_{s}^{2}}\right], \\
\lambda_{-}^{x}=\frac{8}{R^{2}}\left[H_{0}-\frac{2 R^{2}}{\left(R^{2}-\rho_{s}^{2}\right)^{2}}+\frac{2 R^{2}}{\left(R^{2}+\rho_{s}^{2}\right)^{2}}\right],
\end{gathered}
$$

with their respective elastic modes being

$$
\begin{gathered}
s_{1 x}=s_{2 x}=0, \quad s_{1 y}=-s_{2 y}, \quad \rightarrow \text { rotation }, \\
s_{1 x}=s_{2 x}=0, \quad s_{1 y}=+s_{2 y}, \quad \rightarrow \text { translation } \perp, \\
s_{1 x}=+s_{2 x}, \quad s_{1 y}=s_{2 y}=0, \quad \rightarrow \text { translation } \|, \\
s_{1 x}=-s_{2 x}, \quad s_{1 y}=s_{2 y}=0, \quad \rightarrow \text { compression. }
\end{gathered}
$$

In the above expressions, translation $\perp(\|)$ means translation orthogonal (parallel) to the axis joining the two vortices. These elastic modes, as well as $e_{k}=R^{2} \lambda_{ \pm}^{k} / 8 H_{0}$, are plotted in Fig. 5.

It would be interesting to compare the obtained formula for $\lambda_{ \pm}^{i}$ with the ones predicted if there were no interaction with the interface of the disk. The dynamical matrix in such case is much simpler,

$$
\frac{2}{R^{2}} H_{0}\left(\begin{array}{cccc}
3 & -1 & 0 & 0 \\
-1 & 3 & 0 & 0 \\
0 & 0 & 1 & 1 \\
0 & 0 & 1 & 1
\end{array}\right)
$$

which has eigenvalues $0,4 H_{0} / R^{2}$ (twofold degenerate), and $8 H_{0} / R^{2}$, related to pure rotation, translation, and pure compression, respectively. 
*Electronic address: leonardo.cabral@ua.ac.be

†Electronic address: francois.peeters@ua.ac.be

${ }^{1}$ A. Houghton, R. A. Pelcovits, and A. Sudbø, Phys. Rev. B 40, 6763 (1989).

${ }^{2}$ M. V. Feigel'man and V. M. Vinokur, Phys. Rev. B 41, 8986 (1990).

${ }^{3}$ S. Sengupta, C. Dasgupta, H. R. Krishnamurthy, G. I. Menon, and T. V. Ramakrishnan, Phys. Rev. Lett. 67, 3444 (1991).

${ }^{4}$ G. Carneiro, R. Cavalcanti, and A. Gartner, Phys. Rev. B 47, 5263 (1993).

${ }^{5}$ E. Zeldov, D. Majer, M. Konczykowski, V. B. Geshkenbein, V. M. Vinokur, and H. Shtrikman, Nature (London) 375, 373 (1995); D. Majer, E. Zeldov, and M. Konczykowski, Phys. Rev. Lett. 75, 1166 (1995); A. Soibel, Y. Myasoedov, M. L. Rappaport, T. Tamegai, S. S. Banerjee, and E. Zeldov, ibid. 87, 167001 (2001).

${ }^{6}$ H. Pastoriza and P. H. Kes, Phys. Rev. Lett. 75, 3525 (1995).

${ }^{7}$ A. E. Koshelev, Phys. Rev. B 50, 506 (1994).

${ }^{8}$ L. N. Bulaevskii and M. P. Maley, Phys. Rev. Lett. 71, 3541 (1993); L. N. Bulaevskii, J. H. Cho, M. P. Maley, P. Kes, Q. Li, M. Suenaga, and M. Ledvij, Phys. Rev. B 50, 3507 (1994).

${ }^{9}$ E. H. Brandt, Physica C 162-4, 1167 (1989); Int. J. Mod. Phys. B 5, 751 (1991).

${ }^{10}$ E. H. Brandt, J. Low Temp. Phys. 26, 709 (1977).

${ }^{11}$ E. H. Brandt, J. Low Temp. Phys. 26, 735 (1977).

${ }^{12}$ G. Blatter, M. V. Feigel'man, V. B. Geshkenbein, A. I. Larkin, and V. M. Vinokur, Rev. Mod. Phys. 66, 1125 (1994).

${ }^{13}$ E. H. Brandt, Rep. Prog. Phys. 58, 1465 (1995).

${ }^{14}$ A. I. Buzdin and J. P. Brison, Phys. Lett. A 196, 267 (1994).

15 J. J. Palacios, Phys. Rev. B 58, R5948 (1998).

${ }^{16}$ B. J. Baelus, F. M. Peeters, and V. A. Schweigert, Phys. Rev. B 63, 144517 (2001).

${ }^{17}$ B. J. Baelus, L. R. E. Cabral, and F. M. Peeters, Phys. Rev. B 69, 064506 (2004).

${ }^{18}$ L. R. E. Cabral, B. J. Baelus, and F. M. Peeters, Phys. Rev. B 70, 144523 (2004).

${ }^{19}$ V. A. Schweigert and F. M. Peeters, Phys. Rev. B 51, 7700 (1995).

${ }^{20}$ M. Kong, B. Partoens, A. Matulis, and F. M. Peeters, Phys. Rev.
E 69, 036412 (2004).

${ }^{21}$ B. Partoens and P. S. Deo, Phys. Rev. B 69, 245415 (2004).

${ }^{22}$ C. C. de Souza Silva and G. Carneiro, Phys. Rev. B 66, 054514 (2002).

${ }^{23}$ C. C. de Souza Silva, J. A. Aguiar, and V. V. Moshchalkov, Phys. Rev. B 68, 134512 (2003).

${ }^{24}$ A. Buzdin and M. Daumens, Physica C 294, 257 (1998); 332, $108(2000)$

${ }^{25}$ E. H. Brandt and J. R. Clem, Phys. Rev. B 69, 184509 (2004).

${ }^{26}$ B. Partoens and F. M. Peeters, J. Phys.: Condens. Matter 9, 5383 (1997).

${ }^{27}$ Y. J. Lai and L. I, Phys. Rev. E 60, 4743 (1999).

${ }^{28}$ C. P. Bean and J. D. Livingston, Phys. Rev. Lett. 12, 14 (1964).

${ }^{29}$ J. Bardeen and M. J. Stephen, Phys. Rev. 140, A1197 (1965).

${ }^{30}$ D. Reefman and H. B. Brom, Physica C 183, 212 (1991).

${ }^{31}$ C. C. de Souza Silva, L. R. E. Cabral, and J. A. Aguiar, Phys. Rev. B 63, 134526 (2001).

${ }^{32}$ For example, in Ref. 18 we studied a disk of radius $50 \xi$ and compared the results obtained within the GL and London theories, finding that the configurations obtained from the latter approach are stable within the GL theory up to $H_{0}=0.2$. Moreover, the ground-state configurations were the same for the $L$ $=1, \ldots, 9$ states.

${ }^{33}$ For $R=50, h=10$ corresponds to $H_{0}=0.008$. At such $H_{0}, L=4$ is the ground state in both the GL and the London theories (Ref. 18).

${ }^{34}$ V. A. Schweigert and F. M. Peeters, Phys. Rev. Lett. 83, 2409 (1999).

${ }^{35}$ It is worth mentioning that, for $L<9$, the only other configuration which shows a change in the geometry before vortex expulsion was the $L=(6)$ state. In the others a vortex is expelled when the configuration turns out to be a saddle point.

${ }^{36}$ V. M. Bedanov and F. M. Peeters, Phys. Rev. B 49, 2667 (1994).

${ }^{37}$ I. V. Schweigert, V. A. Schweigert, and F. M. Peeters, Phys. Rev. Lett. 84, 4381 (2000).

${ }^{38}$ M. Kong, B. Partoens, and F. M. Peeters, Phys. Rev. E 67, 021608 (2003).

${ }^{39}$ A. Melzer, Phys. Rev. E 67, 016411 (2003).

${ }^{40}$ M.-C. Miguel and S. Zapperi, Nat. Mater. 2, 477 (2003). 